\title{
Linear optimal noncausal control of wave energy converters
}

\author{
Siyuan Zhan and Guang Li*, Member, IEEE
}

\begin{abstract}
This paper addresses the fundamental theoretical development of a linear optimal noncausal control for wave energy converters (WECs) in a closed analytic form. It is well known that wave energy converter control is a noncausal control problem, i.e. the future wave information contributes to the present control action. This paper provides a reliable, efficient and simple linear optimal controller with guaranteed stability for WEC control problem. The proposed WEC linear optimal control (LOC) consists of a causal linear state feedback part and an anticausal linear feedforward part to incorporate the influence of future incoming waves. The stability of the closed-loop WEC control system with the proposed linear optimal controller is proven. The contribution of the noncausal term using wave prediction information to the optimal control and the energy output is analyzed quantitatively. The proposed linear controller can be more preferred when constraint satisfaction becomes a less important issue for mild sea states and some well-designed WECs with an ample operation range. The proposed optimal control strategy can be extended for a generic class of energy maximization problems. Numerical simulations are presented to justify the efficacy of the proposed WEC optimal control.
\end{abstract}

Index Terms-Optimal control, sea wave energy, wave energy converter, energy maximization control, sea wave prediction.

\section{INTRODUCTION}

A Substantial amount of renewable energy is contained in ocean waves. The subject of harnessing wave energy has been extensively studied in recent decades, especially after the oil crisis in 1973 [1], and many different types of wave energy converters (WECs) have been invented since then. However, the WEC technology is still immature compared with other renewable energies, such as wind energy and solar energy [2].

It is well known that control plays an important role in increasing the energy conversion efficiency of WECs. Conventional WEC control strategies are based on the impedance matching principle that energy output can be maximized when the resonance frequency of a WEC matches the dominant frequency of the incoming waves [3]. The typical conventional WEC control strategies include latching control [4], [5], phase control [6] and declutching control [7]. However most of these control strategies are only effective for regular waves and become very complicated to implement for irregular waves in real sea conditions.

Optimal control of WECs is essentially different from the conventional optimal control problems. Firstly, a WEC controller aims to maximize energy output, whereas conventional optimal control has been developed for reference

S. Zhan and G. Li are with the Department of Engineering and Material Science, Queen Mary University of London, London, E1 4NS UK e-mail: (s.zhan,g.li)@qmul.ac.uk.

*Address all correspondence to this author. tracking or equilibrium regulation problems. Because a WEC is subject to the persistent disturbance (excitation force from incoming waves), there are no equilibrium or fixed references for tracking. Secondly, it has been proven that to achieve an optimal operation of a WEC, the future information of the incoming wave is needed for the implementation of a WEC controller, which results in a noncausal optimal control problem [3]. These differences invalidate a direct application of the conventional optimal control strategies.

Some linear causal sub-optimal control strategies have been recently developed for the WEC control problem, e.g. a non-standard linear quadratic Gaussian (LQG) control [8] and the noncausal control proposed in [9]. Although the implementation of these causal optimal controllers avoids the hardware cost for wave prediction, this extra hardware cost can be trivial compared with the increased energy output gained by incorporating wave prediction into control in some scenarios. In [10], we demonstrate that the noncausal optimal WEC control with wave prediction based on the concept of model predictive control (MPC) can at least double the energy output than the energy converted by the traditional causal WEC control methods. Recent progress in wave prediction techniques, e.g. the deterministic sea wave prediction (DSWP) technology developed in [11], makes the employment of wave prediction in a WEC control system more economically viable.

In this paper, we develop a generic linear noncausal optimal control strategy for the WEC control problem. Compared with the existing causal sub-optimal controllers, this controller can explicitly incorporate wave prediction information into the WEC control problem. At the price of not explicitly incorporating constraints into WEC optimal control problem, the resulting optimal controller takes a compact closed analytic form, which provides theoretical insights into WEC optimal control. The control signal is composed of two terms: one feedback term determined by the current measurement of state, and one feedforward term incorporating the influence of current and future waves. The feedback and feedforward gains used to calculate the feedback and feedforward control signals can be efficiently determined off-line. The stability of the proposed noncausal optimal control is also analyzed, which provides a theoretical basis towards the stability analysis of MPC of WECs when constraints are active. The reliable, efficient and simple linear optimal controller with guaranteed stability can be more preferred when the potential constraints violation becomes a less significant issue for mild sea states and some well-designed WECs with an ample operation range, e.g. the M4 device reported in [12]. As an alternative simple controller to MPC, the proposed linear controller can be easily 
tuned and implemented on economically-viable hardware. The proposed optimal control strategy can be extended to cope with a generic class of direct energy output maximization problems where optimal references are difficult to be calculated, or even do not exist, e.g. solar energy and wind energy control problems. This method has more potential especially when the energy source can be predicted.

Although the control method is generic and can be used for a wide variety of WECs, we select a particular WEC as a case study for demonstration purpose. The device to be investigated in this paper reflects the working principle of a point absorber whose schematic diagram is shown in Fig. 1. To present the WEC control problem, we use the models similar to those used in [13]. The excitation wave force drives the float, resulting in the relative heave motion between the piston fixed to the buoy and the cylinder fixed to the seabed (or anchored through mooring lines, but with a negligible heave motion of anti-heave plate compared to that of the float). This relative motion creates fluid flow that drives the power take-off (PTO) system to generate power; the typical PTO mechanism includes direct linear generators [14] and hydraulic motors connected to electricity generators [15].

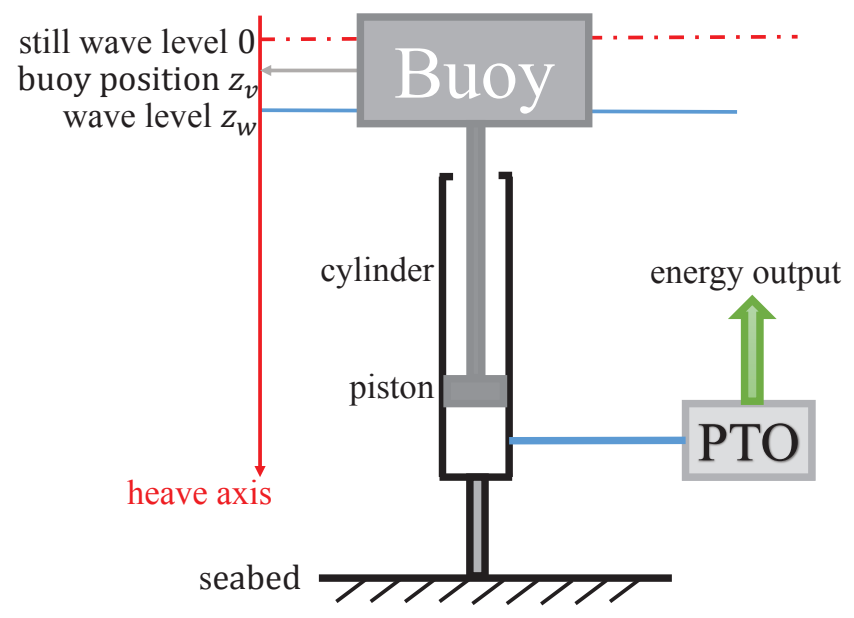

Fig. 1. Schematic diagram of a point absorber

The external manipulated force $f_{u}$ acting on the piston is the control input. $z_{w}$ and $z_{v}$ represent the sea surface level and the heave position of the middle point of the buoy respectively. By defining the direction as shown in Fig. 2, the power output of the WEC at time $t$ is expressed as

$$
P(t)=-f_{u} \dot{z}_{v}
$$

The energy absorbed during period $\left[T_{1}, T_{2}\right]$ is expressed as

$$
\int_{T_{1}}^{T_{2}} P(t) d t
$$

For safety operation, we restrict the float's heave motion so that the constraint can be expressed as

$$
\left|z_{v}\right| \leq \Phi_{\max }
$$

where $\Phi_{\max }$ is the float heave motion limits. The WEC is also subject to control input limitation

$$
\left|f_{u}\right| \leq u_{\max }
$$

The controller design objective is to maximize the energy (1b) subject to the state constraint (2a) and input constraint (2b).

Since linear optimal control cannot explicitly incorporate hard constraints into optimization, we use soft constraints instead. The WEC LOC problem is solved via the Bellman principle [16]. The implementation of the WEC LOC is based on the assumption that full state information is available from a state observer.

The rest of the paper is organized as follows. The dynamic modeling of a single WEC device is presented in Section II. Section III provides the WEC optimal control formulation. Simulation results are demonstrated in Section IV. Finally, the paper is concluded in Section V.

\section{MOdEL SETUP}

The free body diagram of the float is shown in Fig. 2. By

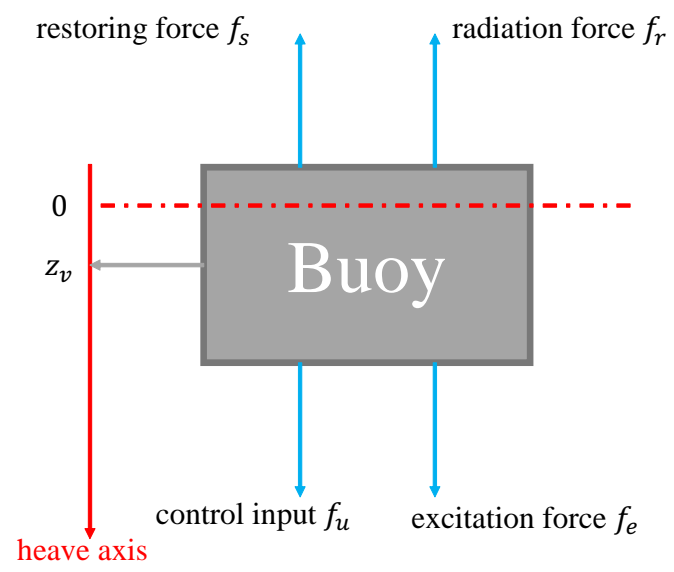

Fig. 2. Free body diagram of the float

applying Newton's second law, we have

$$
m_{s} \ddot{z}_{v}=-f_{s}-f_{r}+f_{e}+f_{u}
$$

where $m_{s}$ is the float mass; the restoring force $f_{s}$ is given by

$$
f_{s}=k_{s} z_{v}
$$

with the hydrostatic stiffness $k_{s}=\rho g s$, and $\rho$ as water density, $g$ as standard gravity, and $s$ as the cross-sectional area of the float. $f_{r}$ is the radiation force determined by

$$
f_{r}=m_{\infty} \ddot{z}_{v}+\int_{-\infty}^{\infty} h_{r}(\tau) \dot{z}_{v}(t-\tau) d \tau
$$

where $m_{\infty}$ is the added mass; $h_{r}$ is the kernel of the radiation force that can be computed via hydraulic software packages (e.g. WAMIT [17]). Following [13], the convolutional term in (5) $f_{R}:=\int_{-\infty}^{\infty} h_{r}(\tau) \dot{z}_{v}(t-\tau) d \tau$ can be approximated by a causal finite dimensional state-space model

$$
\begin{aligned}
& \dot{x}_{r}=A_{r} x_{r}+B_{r} \dot{z}_{v} \\
& f_{r}=C_{r} x_{r} \approx \int_{-\infty}^{t} h_{r}(\tau) \dot{z}_{v}(t-\tau) d \tau
\end{aligned}
$$


where $\left(A_{r}, B_{r} C_{r}, 0\right)$ and $x_{r} \in \mathbb{R}^{n_{r}}$ are the state-space realisation and the state respectively. Following [13], the wave excitation force $f_{e}$ can be determined by

$$
f_{e}=\int_{-\infty}^{\infty} h_{e}(\tau) z_{w}(t-\tau) d \tau
$$

where $h_{e}$ is the kernel of the radiation force and the state-space approximation is given by

$$
\begin{aligned}
& \dot{x}_{e}=A_{e} x_{e}+B_{e} z_{w} \\
& f_{e}=C_{e} x_{e} \approx \int_{-\infty}^{t} h_{e}(\tau) z_{w}(t-\tau) d \tau
\end{aligned}
$$

where $\left(A_{e}, B_{e} C_{e}, 0\right)$ and $x_{e} \in \mathbb{R}^{n_{e}}$ are the state-space realization and the state respectively.

With the realizations of (6) and (8a), the state-space model of (3) can be represented by

$$
\left\{\begin{array}{l}
\dot{x}=A_{c} x+B_{u c} u+B_{w c} w \\
z=C_{z} x
\end{array}\right.
$$

where $w:=z_{w}, z:=z_{v}, y:=\dot{z}_{v}, x:=\left[z_{v}, \dot{z}_{v}, x_{r}, x_{e}\right]$ and

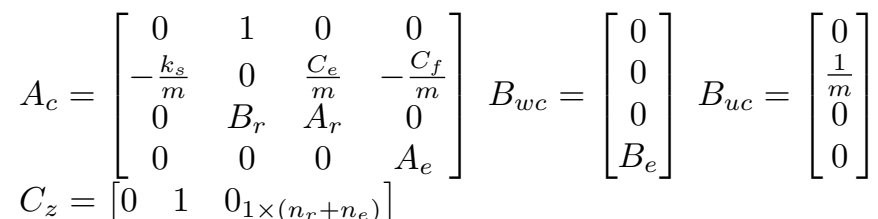

with $m:=m_{a}+m_{\infty}$.

The continuous time model (9) can be converted to a discrete time model

$$
\left\{\begin{array}{l}
x_{k+1}=A x_{k}+B_{u} u_{k}+B_{w} w_{k} \\
z_{k}=C_{z} x_{k}
\end{array}\right.
$$

The WEC linear optimal control development in the remaining paper will be based on this discrete time model.

\section{WEC OPTIMAL CONTROL FORMULATION}

\section{A. Optimal controller derivation of WEC}

The optimal control strategy of a WEC can be written as

$$
\begin{aligned}
& \min _{u} \frac{1}{N}\left(\sum_{k=0}^{N-1} L_{k}\left(x_{k}, u_{k}\right)\right), \\
& \text { s. t. } x_{k+1}=A x_{k}+B_{u} u_{k}+B_{w} w_{k} \\
& \quad z_{k}=C_{z} x_{k}
\end{aligned}
$$

where $N$ is the number of prediction steps and $L_{k}$ is a stage cost

$$
L_{k}=\frac{1}{2} x_{k}^{T} Q x_{k}+z_{k} u_{k}+\frac{1}{2} r u_{k}^{2}
$$

Here

- $\frac{1}{2} x_{k}^{T} Q x_{k}$ is used to penalize the state. The weight $Q$ influences the stability of the control system and can be used as a tuning parameter to handle the state constraint (2a).

- $-z_{k} u_{k}$ represents the power that can be captured by the power take-off (PTO) mechanism, i.e. minimizing $z_{k} u_{k}$ is equivalent to maximizing the energy output.
- $\frac{1}{2} r u_{k}^{2}$ is used to penalize the input. The weight $r$ influences the stability of the control system and can be used as a tuning parameter to handle the input constraint (2b).

Remark 1. If the constraints (2a) and (2b) are not explicitly included in the optimization, an analytical solution can be derived. The weights $Q$ and $R$ can be used to penalize $x$ and $u$ in the objective function and can be used as the tuning parameters to avoid constraint violations.

The linear optimal noncausal control policy for WEC control is given in the following Theorem:

Theorem 1. The linear optimal noncausal controller for the control problem (12) is

$$
u_{k}=K_{x, k} x_{k}+K_{w, k} w_{k}+K_{s, k} s_{k+1}
$$

where

$$
\begin{aligned}
K_{x, k} & =-\left(r+B_{u}^{T} V_{k+1} B_{u}\right)^{-1}\left(C_{z}+B_{u}^{T} V_{k+1} A\right) \\
K_{w, k} & =-\left(r+B_{u}^{T} V_{k+1} B_{u}\right)^{-1} B_{u}^{T} V_{k+1} B_{w} \\
K_{s, k} & =-\left(r+B_{u}^{T} V_{k+1} B_{u}\right)^{-1} B_{u}^{T}
\end{aligned}
$$

and $V_{k}$ and $s_{k+1}$ can be calculated via backward iterations

$$
\begin{aligned}
V_{k}= & Q_{k}+A^{T} V_{k+1} A-\left(C_{z}+B_{u}^{T} V_{k+1} A\right)^{T}(r \\
& \left.+B_{u}^{T} V_{k+1} B_{u}\right)^{-1}\left(C_{z}+B_{u}^{T} V_{k+1} A\right) \\
s_{k}= & \left(A+B_{u} K_{x, k}\right)^{T}\left(V_{k+1} B_{w} w_{k}+s_{k+1}\right)
\end{aligned}
$$

with the boundary conditions

$$
\begin{aligned}
& V_{N}=0 \\
& s_{N}=0
\end{aligned}
$$

Proof. Define the optimal cost-to-go (from $k$ to $N$ ) to be $v(x, k)$. Since the dynamics are linear and the stage cost are quadratic, a reasonable guess of the cost-to-go function can be quadratic on the current state $x_{k}$

$$
v(x, k)=\frac{1}{2} x_{k}^{T} V_{k} x_{k}+x_{k}^{T} s_{k}+a_{k}
$$

where $V_{k}, s_{k}, a_{k}$ do not depend on $x_{k}$ and $V_{k} \geq 0$. From

$$
v(x, N)=\frac{1}{2} x_{N}^{T} V_{N} x_{N}+x_{N}^{T} s_{N}+a_{N}=0
$$

we have the boundary conditions $V_{N}=0, s_{N}=0, a_{N}=0$ for any $x_{N}$. From the Bellman optimality principle [16], we have

$$
v(x, k)=\min _{u_{k}}\left\{L\left(x_{k}, u_{k}\right)+v(x, k+1)\right\}
$$

Define $P\left(x_{k}, u_{k}, w_{k}\right):=L\left(x_{k}, u_{k}\right)+v(x, k+1)$. Since $\frac{\partial^{2} P\left(x_{k}, u_{k}, w_{k}\right)}{\partial u_{k}^{2}}=r+B_{u}^{T} V_{k+1} B_{u}>0$, we have

$$
\begin{aligned}
u_{k}= & \frac{\partial P\left(x_{k}, u_{k}, w_{k}\right)}{\partial u_{k}} \\
= & -\left(r+B_{u}^{T} V_{k+1} B_{u}\right)^{-1}\left[\left(B_{u}^{T} V_{k+1} A+C_{z}\right) x_{k}\right. \\
& \left.+B_{u}^{T} V_{k+1} B_{w} w_{k}+B_{u}^{T} s_{k+1}\right]
\end{aligned}
$$


which results in (13). Then we replace $u_{k}$ in (16c) with (13). Since (16c) needs to be satisfied for all $x_{k}$, after some calculation and rearrangement, we have

$$
\begin{gathered}
V_{k}=Q+A^{T} V_{k+1} A-\left(C_{z}+B_{u}^{T} V_{k+1} A\right)^{T}(r \\
\left.+B_{u}^{T} V_{k+1} B_{u}\right)^{-1}\left(C_{z}+B_{u}^{T} V_{k+1} A\right) \\
s_{k}=\left(A+B_{u} K_{x, k}\right)^{T}\left(V_{k+1} B_{w} w_{k}+s_{k+1}\right)
\end{gathered}
$$

and $a_{k}$ satisfies

$$
a_{k}=a_{k+1}+M_{1}-M_{2}+M_{3}
$$

where

$$
\begin{aligned}
M_{1}= & \frac{1}{2}\left(B_{w} w_{k}\right)^{T} V_{k+1}\left(B_{w} w_{k}\right) \\
M_{2}= & \frac{1}{2}\left(K_{w, k} w_{k}+K_{s, k} s_{k+1}\right)^{T}\left(B_{u}^{T} V_{k+1} B_{u}\right. \\
& +r)\left(K_{w, k} w_{k}+K_{s, k} s_{k+1}\right) \\
M_{3}= & w_{k}^{T} B_{w}^{T} s_{k+1}
\end{aligned}
$$

which completes the proof.

Remark 2. From Theorem 1, we can see that the optimal control consists of two parts:

- the causal linear part $K_{x, k} x_{k}+K_{w, k} w_{k}$, i.e. the part depending on current state and wave information.

- the anticausal part $K_{s, k} s_{k+1}$, where $s_{k+1}$ depends only on the future wave information provided by some wave prediction technique. This enables one to quantitatively investigate the influence of anticausal term on energy output.

The coefficients $K_{x, k}, K_{w, k}$ and $K_{s, k}$ can be determined offline.

Remark 3. The calculation of the optimal controller (13) can be partially computed offline for the gains $K_{x, k}, K_{w, k}$ and $K_{s, k}$ and partially computed online for $s_{k}$ incorporating the wave prediction information. The control algorithm is shown in Algorithm 1.

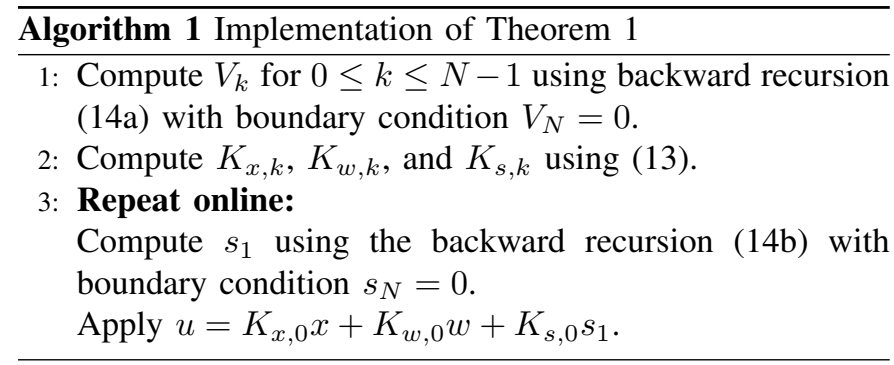

Note that the term $s_{1}$ calculated from the online backward recursion can be viewed as the accumulation of the impact from the incoming wave prediction on the optimal control action.

\section{B. Steady-state solution}

In this section, we develop a steady state solution so that the stability can be proved and Algorithm 1 can be further simplified for implementation. We start with the backward recursion of the discrete-time Ricatti equation (14a).
Assumption 1. The system $\left(A, B_{u}\right)$ is stabilizable. The coefficients of the cost function are chosen such that $\left[\begin{array}{cc}Q & C_{z}^{T} \\ C_{z} & r\end{array}\right]>$ 0 . The control horizon is infinite, i.e. $N \rightarrow \infty$ in the cost function (12a).

Lemma 1 (Convergence \& Stability). If Assumption 1 holds, the solution of the Ricatti equation (14a) converges as $N \rightarrow$ $\infty$, i.e. $V_{0} \rightarrow V$ and $V$ satisfies the discrete algebraic Ricatti equation $(D A R E)$

$$
\begin{aligned}
V= & A^{T} V A+Q \\
& -\left(B_{u}^{T} V A+C_{z}\right)^{T}\left(r+B_{u}^{T} V B_{u}\right)^{-1}\left(B_{u}^{T} V A+C_{z}\right)
\end{aligned}
$$

and the solution of the DARE (17) is unique. The control action to be implemented can be simplified as

$$
u=K_{x} x+K_{w} w+K_{s} s_{1}
$$

where

$$
\begin{aligned}
K_{x} & =-\left(r+B_{u}^{T} V B_{u}\right)^{-1}\left(C_{z}+B_{u}^{T} V A\right) \\
K_{w} & =-\left(r+B_{u}^{T} V B_{u}\right)^{-1} B_{u}^{T} V B_{w} \\
K_{s} & =-\left(r+B_{u}^{T} V B_{u}\right)^{-1} B_{u}^{T}
\end{aligned}
$$

and $s_{1}$ is the solution of the online backward recursion (14b). With this control law, the system is stable, i.e. $A+B_{u} K_{x}$ has eigenvalues strictly inside the unit circle.

Proof. We begin the proof by introducing a nominal system

$$
\bar{x}_{k+1}=A \bar{x}_{k}+B_{u} \bar{u}_{k}
$$

and an optimization problem based on this nominal system

$$
\begin{aligned}
J_{N-k}\left(\bar{x}_{N-k}\right)= & \min _{\bar{u}_{N-k}, \ldots, \bar{u}_{N-1}} \bar{x}_{N}^{T} Q_{N} \bar{x}_{N} \\
& +\sum_{i=N-k}^{N-1}\left[\begin{array}{c}
\bar{x}_{i} \\
\bar{u}_{i}
\end{array}\right]^{T}\left[\begin{array}{cc}
Q & C_{z}^{T} \\
C_{z} & r
\end{array}\right]\left[\begin{array}{l}
\bar{x}_{i} \\
\bar{u}_{i}
\end{array}\right]
\end{aligned}
$$

By assuming $J_{k}\left(\bar{x}_{N-k}\right)=\bar{x}_{N-k}^{T} \bar{V}_{N-k} x_{N-k}$ where $\bar{V}_{N-k} \geq$ 0 and with the Bellman optimality principle [16], we have

$$
\begin{aligned}
J_{N-k}\left(\bar{x}_{N-k}\right)= & \min _{u_{N-k}}\left[J_{N-k+1}\left(\bar{x}_{N-k+1}\right)\right. \\
& \left.+\left[\begin{array}{c}
\bar{x}_{N-k} \\
\bar{u}_{N-k}
\end{array}\right]^{T}\left[\begin{array}{cc}
Q & C_{z}^{T} \\
C_{z} & r
\end{array}\right]\left[\begin{array}{l}
\bar{x}_{N-k} \\
\bar{u}_{N-k}
\end{array}\right]\right]
\end{aligned}
$$

Following the similar method to (14), we have

$$
\begin{aligned}
\bar{V}_{k}= & Q+A^{T} \bar{V}_{k+1} A-\left(C_{z}+B_{u}^{T} \bar{V}_{k+1} A\right)^{T}(r \\
& \left.+B_{u}^{T} \bar{V}_{k+1} B_{u}\right)^{-1}\left(C_{z}+B_{u}^{T} \bar{V}_{k+1} A\right)
\end{aligned}
$$

with boundary condition $\bar{V}_{N}=Q_{N}$, with optimal control action

$$
\bar{u}_{k}^{*}=\bar{K}_{x, k} \bar{x}_{k}
$$

where $\bar{K}_{x, k}-\left(r+B_{u}^{T} \bar{V}_{k+1} B_{u}\right)^{-1}\left(B_{u}^{T} \bar{V}_{k+1} A+C_{z}\right)$

By defining $S_{k}=\bar{V}_{N-k}$, the backward recursion (22) can be rewritten as

$$
\begin{aligned}
S_{k+1}= & Q+A^{T} S_{k} A-\left(C_{z}+B_{u}^{T} S_{k} A\right)^{T}(r \\
& \left.+B_{u}^{T} S_{k} B_{u}\right)^{-1}\left(C_{z}+B_{u}^{T} S_{k} A\right)
\end{aligned}
$$


with $S_{0}=Q_{N}$. With (20) to (24), after rearrangement (replacing $\bar{x}_{N-k}$ with $\bar{x}_{0}$ ), we have

$$
\bar{x}_{0} S_{k} \bar{x}_{0}:=P_{k}^{*}\left(Q_{N}\right)=\min _{u_{0}, \ldots, u_{k-1}} P_{k}\left(Q_{N}\right)
$$

where $P_{k}^{*}\left(Q_{N}\right)$ denotes the optimal value of $P_{k}\left(Q_{N}\right)$ and

$$
P_{k}\left(Q_{N}\right):=x_{k}^{T} Q_{N} x_{k}+\sum_{i=0}^{k-1}\left[\begin{array}{l}
\bar{x}_{i} \\
\bar{u}_{i}
\end{array}\right]^{T}\left[\begin{array}{cc}
Q & C_{z}^{T} \\
C_{z} & r
\end{array}\right]\left[\begin{array}{l}
\bar{x}_{i} \\
\bar{u}_{i}
\end{array}\right]
$$

Then we move to the main part of the proof.

1) Convergence:

By assuming $Q_{N}=0$, (22) becomes identical with (16d). Since $\left(A, B_{u}\right)$ is stabilizable, there exists a feedback control sequence $M_{k}$ such that the state $x_{0}$ can be regulated to the origin in $n_{x}$ steps, where $n_{x}$ is the dimension of the state $x_{k}$, with control sequence $u_{k}=M_{k} x_{k}, P_{k}(0)=c_{1}$ for $k>n_{x}$, where $c_{1}$ is a constant.

Meanwhile, since $\left[\begin{array}{cc}Q & C_{z}^{T} \\ C_{z} & r\end{array}\right]>0, P_{k}^{*}(0)$ is increasing, i.e. $P_{k}^{*}(0) \leq P_{k+1}^{*}(0)$. Also, $P_{k}^{*}(0)=\bar{x}_{0}^{T} S_{k} \bar{x}_{0}$ is upper bounded by $P_{k}^{*}(0) \leq P_{k}(0)$, which means $P_{k}^{*}(0)$ is converging, i.e. $\lim _{n \rightarrow \infty} P_{k}^{*}(0)=P_{\infty}^{*}(0)$.

Since the above holds for all $\bar{x}_{0}$, both the solution of (24) with $Q_{N}=0$ and (16d) are converging, i.e. $V_{0}=S_{\infty} \rightarrow V$ as $N \rightarrow \infty$ and can be calculated by (17) and (13) converges to (18a).

2) Stability:

From $\lim _{k \rightarrow \infty} P_{k}^{*}(0)=P_{\infty}^{*}(0)$, we have

$$
\lim _{k \rightarrow \infty}\left[\begin{array}{c}
\bar{x}_{k} \\
\bar{u}_{k}
\end{array}\right]^{T}\left[\begin{array}{cc}
Q & C_{z}^{T} \\
C_{z} & r
\end{array}\right]\left[\begin{array}{c}
\bar{x}_{k} \\
\bar{u}_{k}
\end{array}\right]=0
$$

Since $\left[\begin{array}{cc}Q & C_{z}^{T} \\ C_{z} & r\end{array}\right]>0$, we have $\lim _{k \rightarrow \infty} \bar{u}_{k}=0$ and $\lim _{k \rightarrow \infty} \bar{x}_{k}=0$. The system (19) with optimal control action $\bar{u}^{*}=\lim _{k \rightarrow \infty} \bar{K}_{x, k} \bar{x}=K_{x} \bar{x}$ with $K_{x}$ defined in (18a) is stable. Then we have $A+B_{u} K_{x}$ has all eigenvalues strictly inside the unit circle.

3) Uniqueness of solution:

Assume $u_{k}^{*}$ is the corresponding optimal control action of $P_{k}^{*}(0)$ and $Q_{N}$ is an arbitrary positive semi-definite function. Immediately, we have

$$
P_{k}^{*}(0) \leq P_{k}^{*}\left(Q_{N}\right) \leq P_{k}\left(Q_{N}\right)
$$

By assuming $P_{k}\left(Q_{N}\right)$ using the control action $u_{k}^{*}$, we have $P_{k}\left(Q_{N}\right)=P_{k}^{*}(0)+\bar{x}_{k}^{T} Q_{N} \bar{x}_{k}^{T}$. Since $\lim _{k \rightarrow \infty} \bar{x}_{k}=0$, we have

$$
\begin{aligned}
\lim _{k \rightarrow \infty} P_{k}\left(Q_{N}\right) & =\lim _{k \rightarrow \infty} P_{k}^{*}(0)+\lim _{k \rightarrow \infty} \bar{x}_{k}^{T} Q_{N} \bar{x}_{k}^{T} \\
& =\lim _{k \rightarrow \infty} P_{k}^{*}(0)=P_{\infty}^{*}(0)
\end{aligned}
$$

Combining (26) and (27), we have

$$
\lim _{k \rightarrow \infty} P_{k}^{*}(0)=\lim _{k \rightarrow \infty} P_{k}^{*}\left(Q_{N}\right)=P_{\infty}^{*}(0)
$$

Assume $\tilde{V}$ is another solution of (17) other than $V$. Then we have

$$
\lim _{k \rightarrow \infty} P_{k}^{*}(\tilde{V})=\bar{x}_{0}^{T} \tilde{V} \bar{x}_{0}
$$

We also have

$$
\lim _{k \rightarrow \infty} P_{k}^{*}(\tilde{V})=\lim _{k \rightarrow \infty} P_{k}^{*}(\tilde{0})=\bar{x}_{0}^{T} V \bar{x}_{0}
$$

With (29) and (30), we have $\tilde{V}=V$, i.e. the solution of DARE (17) is unique.

Remark 4. The control law proposed in Lemma 1 is practically intractable because solving $s_{1}$ in (18a) using backward recursion (14b) requires infinite wave prediction horizon as $N \rightarrow \infty$. However, only finite length of wave prediction is achievable from the wave prediction technique.

Remark 5. Recent studies [18], [19] show that the requirement for the stage cost $\left[\begin{array}{cc}Q & C_{z}^{T} \\ C_{z} & r\end{array}\right]$ to be positive definiteness can be relaxed, so that greater weight can be imposed on the energy output term and the DARE still yields a unique stabilizing solution.

To make the control law proposed in Lemma 1 practically implementable, in the remaining of the section, we will show that if the wave prediction horizon is finite but long enough, the control law proposed in Lemma 1 can be further simplified.

Assumption 2. The wave prediction technique can provide sufficiently long $n_{p}$ steps of future wave prediction.

Lemma 2. If Assumption 1 and 2 hold, the control law proposed in Lemma (1) can be further simplified by linear optimal noncausal control law

$$
u=K_{x} x+K_{d} \mathbf{w}
$$

where

$$
\begin{aligned}
K_{x}= & -\left(r+B_{u}^{T} V B_{u}\right)^{-1}\left(C_{z}+B_{u}^{T} V A\right) \\
K_{d}= & -\left(r+B_{u}^{T} V B_{u}\right)^{-1} B_{u}^{T} \Psi \\
V= & A^{T} V A+Q \\
& -\left(B_{u}^{T} V A+C_{z}\right)^{T}\left(r+B_{u}^{T} V B_{u}\right)^{-1}\left(B_{u}^{T} V A+C_{z}\right)
\end{aligned}
$$

$x$ is the current state; $\mathbf{w}:=\left[w_{0}, w_{1}, \ldots, w_{n_{p}-1}\right]^{T}$ is the sequence of predicted future wave heave velocities; $\Phi=$ $\left(A+B_{u} K_{x}\right)^{T} ; \Psi:=\left[V B_{w}, \Phi V B_{w}, \ldots, \Phi^{n_{p}-1} V B_{w}\right]$.

Proof. From Lemma 1, as $N \rightarrow \infty$, the coefficients $K_{x, k}$ and $V_{k+1}$ converge for $0 \leq k \leq n_{p}-1$, i.e. $K_{x, k}=K_{x}, V_{k+1}=$ $V$. The backward recursion of $s_{k}(16 \mathrm{e})$ can be rewritten in a compact form

$$
s_{k}=\Phi s_{k+1}+\Phi V B_{w} w_{k}
$$

where $\Phi=\left(A+B_{u} K_{x}\right)^{T}$.

Define a sequence of $n_{p}$ steps of predicted wave heave elevation profile as $\mathbf{w}:=\left[w_{0}, w_{1}, \ldots, w_{n_{p}-1}\right]^{T}$. The backward recursion (32) can be represented by

$$
s_{1}=\Phi^{n_{p-1}} s_{n_{p}}+\left[0, \Phi V B_{w}, \ldots, \Phi^{n_{p}-1} V B_{w}\right] \mathbf{w}
$$


Substituting (33) into (18a) gives

$$
\begin{aligned}
u & =K_{x} x+K_{s} s_{1}+K_{w} w_{0} \\
& =K_{x} x+K_{s} \Phi^{n_{p-1}} s_{n_{p}}+K_{d} \mathbf{w}
\end{aligned}
$$

where $K_{d}, K_{x}$ and $\Phi$ are defined in (31).

Note that since $\Phi$ has all the eigenvalues strictly inside the unit circle, the impact of future waves on the control input diminishes as the power of $\Phi$ increases. This implies that the wave prediction of near future has more significant impact on control actions than that of the far future, and thus justifies that the term $K_{s} \Phi^{n_{p}-1} s_{n_{p}}$ in (34) can be negligible with sufficient large wave prediction horizon $n_{p}$ in real time implementation. Then we have (31), which completes the proof.

\section{Sea wave prediction techniques}

The efficacy of wave prediction techniques plays a key role in the proposed noncausal optimal WEC control strategy. Different wave prediction techniques have been developed, which can be generally classified into two categories. One class of wave prediction techniques are based on the past sea wave data measured at the same point of the sea surface where the WEC is located. This class of methods for the prediction are based on statistical methods, such as autoregressive models, cyclical models, extended Kalman filters, as summarized in [20]. The other class of techniques are based on the measurements of sea wave elevations at multiple nearby locations with certain distances away from the WEC. The information of wave propagation and wave directions can be used to predict the sea wave profile at the WEC's location with tens of seconds into the future. A representative wave prediction method in this category is the deterministic sea wave prediction (DSWP) method. We briefly describe its principle for completeness, and more details can be found in [11] and the references therein. Because of the requirement of fast real-time wave prediction in the linear noncausal optimal control for WECs, we use a standard linear oceanographic wave model [21], [22]. The weight elevation at the spatial coordinates $\left(p_{x}, p_{y}\right)$ at time $t$ has the form of

$$
\begin{aligned}
h\left(p_{x}, p_{y}, t\right)= & \sum_{n=1}^{N_{p}} \sum_{r=1}^{R_{s}} \mathcal{A}\left(\omega_{n}, \theta_{r}\right) \cos \left[k_{n} p_{x} \cos \left(\theta_{r}\right)\right. \\
& \left.+k_{n} p_{y} \sin \left(\theta_{r}\right)-\omega_{n} t+\Theta\left(\omega_{n}, \theta_{r}\right)\right]
\end{aligned}
$$

where $N_{p}$ is the number of frequencies employed; $R_{s}$ is the number of significant storm directions which can be assumed to be modest, i.e. $R_{s}<10 ; k_{n}$ is the wave number, which is computed via $k_{n}=2 \pi / \lambda_{n}$, where $\lambda_{n}$ is the wave length; $\mathcal{A}\left(\omega_{n}, \theta_{r}\right)$ is the directional magnitude spectrum, where $\theta_{r}$ is the propagation direction of an individual wave component and $\omega_{n}$ is the angular frequency; $\Theta\left(\omega_{n}, \theta_{r}\right)$ is a phase-lift. The principle of DSWP method is to use the wave data collected from the remote wave sensors at multiple fixed positions to estimate the parameters in wave model (35) and use the model to predict the future wave profile.

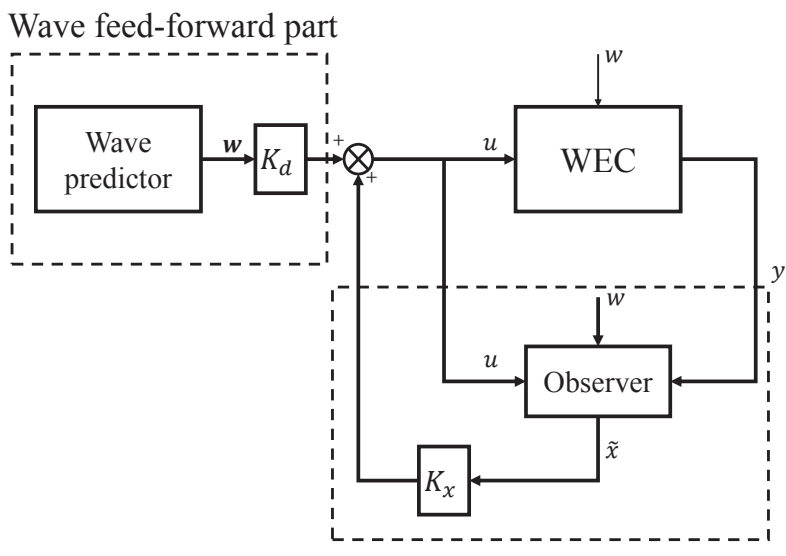

Feedback part

Fig. 3. WEC linear optimal noncausal control framework

\section{Implementation}

In previous sections, we have designed the WEC optimal control based on the assumption that full state information is available. However, in practice it may not always be realistic or economically viable to measure all the states of a WEC, in which case a state observer is required. We assume that only some outputs can be measured

$$
y(k)=C x(k)+v(k)
$$

where $v$ represents the measurement noise and the pair $(A, C)$ is assumed to be observable. Then a standard Kalman observer can be designed to estimate the full state information of a WEC, see [23] for more details. The WEC optimal control framework is shown in Fig. 3. The future wave profile is predicted (by e.g. DSWP [10], [11]). The control input consists of (i) the anticausal feedforward part from the predicted wave information and (ii) the causal feedback part from the estimated states $\tilde{x}$ provided by a state estimator. The WEC optimal control with estimation can be implemented by Algorithm 2 . Based on the separation principle, the whole system is stable if both the observer and the controller are stable.

\section{NUMERICAL EXAMPLES}

In this section, we demonstrate the efficacy of the proposed methods using two sets of numerical examples based on different WEC designs. The WEC model used in the simulation has the form as described in (10). The optimal control policies for WECs are generated by Algorithm 2.

\section{A. The WEC model with parameters used in [13] and no model mismatch}

The parameters of the WEC model used in section IV-A are adopted from those used in [13], which are summarized in Table I.

After discretizing the model with a sampling time $T_{s}=$ $0.1 \mathrm{~s}$, we formulate an optimal control with the objective 
Algorithm 2 Implementation of WEC linear optimal noncausal control.

1: Wave prediction $\mathbf{w}$ from DSWP.

2: State estimation updates:

$$
\tilde{x}(k \mid k)=\tilde{x}(k \mid k-1)+L(y(k)-C \tilde{x}(k \mid k-1))
$$

where $\tilde{x}(k \mid k)$ represents $\tilde{x}_{k}$ estimated at time $k ; L$ is the Kalman gain which can be computed offline by

$$
L=-P C^{T}\left(C P C+R_{v}\right)^{-1}
$$

where the matrix $P$ is the solution of DARE

$$
\begin{aligned}
P= & A P A^{T}-A P C^{T}\left(C P C^{T}+R_{v}\right)^{-1} C P A^{T} \\
& +B_{w} R_{w} B_{w}^{T}
\end{aligned}
$$

where $R_{v}$ and $R_{w}$ are weighting matrices and are tuned to be according to the estimated covariance of measurement noise and incoming wave respectively.

3: Control action update:

$$
u(k \mid k)=K_{x} \tilde{x}(k \mid k)+K_{d} \mathbf{w}
$$

where $K_{x}$ and $K_{d}$ are derived from Lemma 2.

4: Estimator updates:

$$
\tilde{x}(k+1 \mid k)=A \tilde{x}(k \mid k)+B_{u} u(k \mid k)+B_{w} w
$$

TABLE I

THE PARAMETERS USED FOR THE WEC MODEL IN SIMULATION SET A

\begin{tabular}{lll}
\hline Description & Notation & values \\
\hline Stiffness & $k_{s}$ & $3866 \mathrm{~N} / \mathrm{m}$ \\
Float mass & $m_{s}$ & $242 \mathrm{~kg}$ \\
Added mass & $m_{a}$ & $83.5 \mathrm{~kg}$ \\
Total mass & $m$ & $325.5 \mathrm{~kg}$ \\
Input force limit & $u_{\max }$ & $8 \mathrm{kN}$ \\
Float heave limit & $\Phi_{\max }$ & $0.5 \mathrm{~m}$ \\
\hline
\end{tabular}

function as described in (12). The weights $Q$ and $r$ are tuned (i) to be sufficiently small for maximizing the energy output; (ii) to guarantee a unique stabilizing solution can be derived from DARE (17); (iii) to ensure input and heave constraints are not violated. Here we choose $Q=\operatorname{diag}(6,9.8,0, \ldots, 0)$, $r=0.08$ respectively. The observer weights are tuned at $R_{v}=0.01, R_{w}=1$ respectively.

Then we move to the formulation of LOC for WEC using the finite wave prediction with the prediction time horizon $t_{p}:=T_{s} n_{p}$ and $n_{p}$ represents the prediction horizon steps. Note that in designing the WEC LOC, the term $K_{s} \Phi^{n_{p}-1} s_{n_{p}}$ described in (34), where $s_{n_{p}}$ summarizes the future wave information for $t>t_{p}$, is neglected in designing the LOC for WEC.

Fig. 4 shows that the closed-loop system is stable, i.e. $\Phi=A+B K_{x}$ has eigenvalues strictly inside the unit circle. With such properties, we can see that the coefficient on $s_{n_{p}}$ is deceasing with the increase of $n_{p}$, which means that the term $K_{s} \Phi^{n_{p}-1} s_{n_{p}}$ can be negligible for a sufficiently large wave prediction horizon.

Fig. 5 shows the change of the gain $K_{d}=$ $\left[K_{d}(0), K_{d}(1), \ldots, K_{d}\left(n_{p}-1\right)\right]$ over the prediction steps.
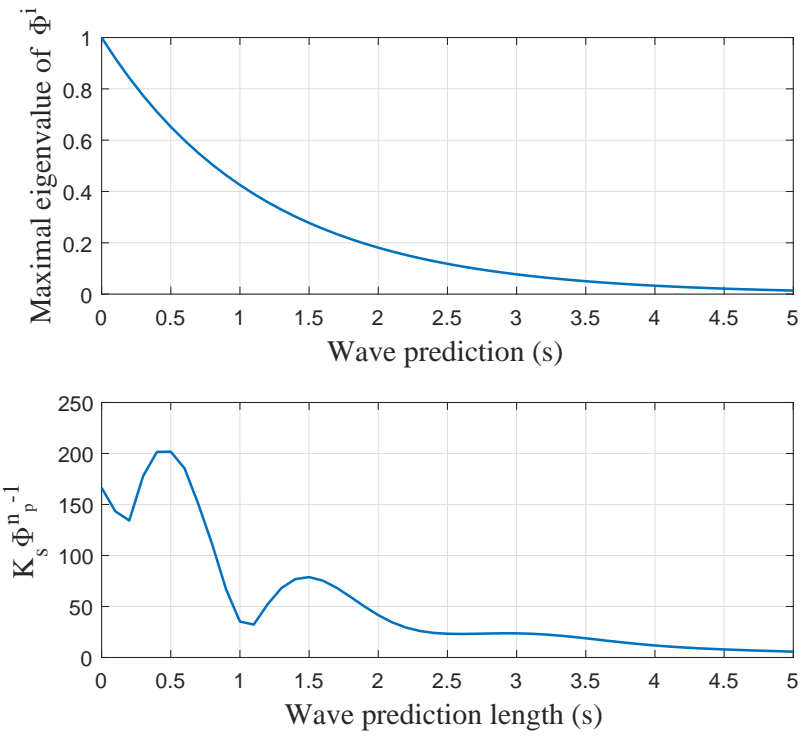

Fig. 4. The coefficient on $s_{n_{p}}$ is decreasing with the increase of $n_{p}$, which means that the term $K_{s} \Phi^{n_{p}-1} s_{n_{p}}$ can be negligible for sufficiently large wave prediction length $n_{p}$.

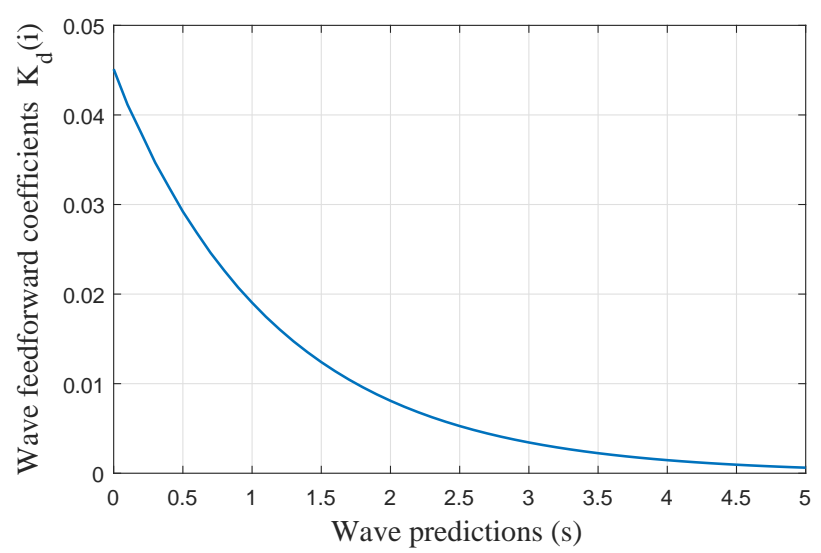

Fig. 5. Impact of future wave predictions on control

Here, $K_{d}(i)$ represents the gain of wave prediction at step $n=i$, i.e. wave prediction at time $t=T_{s} i$. We can see the magnitude of the $K_{d}(i)$ decreases with the increase of prediction step $i$, which means that the impact of future wave prediction decreases with time in wave feedforward control part $K_{d} \mathbf{w}$.

The real wave data gathered off the coast of Cornwall UK are used for numerical simulations. The 50 seconds of the heave elevation used in simulation are shown in Fig. 6.

Four cases with different controllers are calculated with different wave prediction horizons $(0 \mathrm{~s}, 1 \mathrm{~s}, 2 \mathrm{~s}$ and $3 \mathrm{~s})$. Fig. 7 and Fig. 8 show the control inputs $u=K_{x} x+K_{d} \mathbf{w}$ and the wave feedforward part signals $K_{d} \mathbf{w}$ for the four cases respectively. The limit on control input magnitude is $|u| \leq 8$ $\mathrm{kN}$. Note that the LOC with wave prediction horizon of 0 $\mathrm{s}$ is the causal LOC and can be used for comparison with the noncausal optimal controllers proposed in this paper. By comparing the control input without wave prediction (i.e. the 


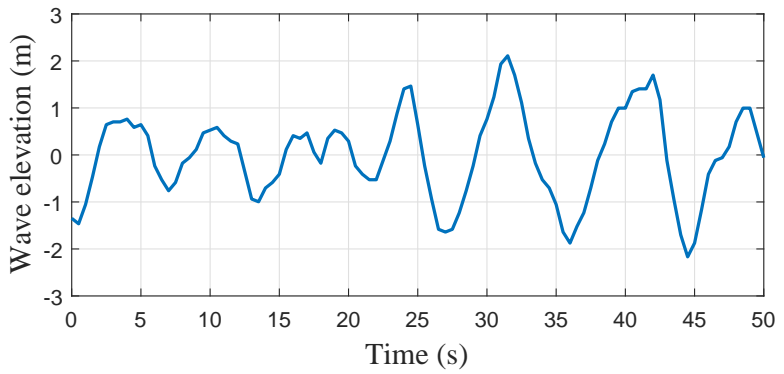

Fig. 6. A 50-second period of wave profile (wave elevation) is used in simulation.

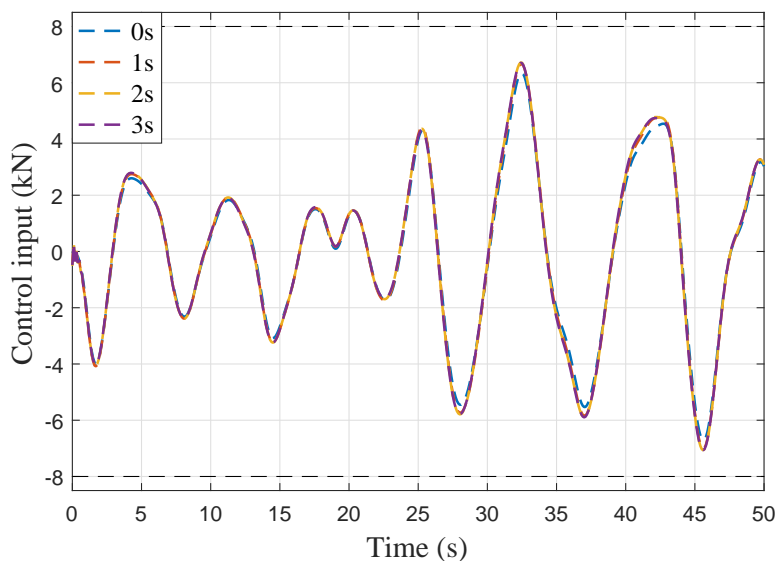

Fig. 7. Control input magnitude with different wave prediction horizons $(0 \mathrm{~s}$ corresponds to no wave prediction). Inclusion of feedward control for wave prediction does not change control input magnitude significantly.

0s line) with other control inputs with wave prediction in Fig. 7 , we can see that the magnitude of maximal control input signal does not significantly change when feedforward term is added. This feature is helpful because it not only provides a fair comparison basis, but also reduces controller design complexity because the WEC control designers do not have to retune the feedback part when adding the wave feedforward part.

Fig. 9 shows buoy heave displacement response of the WEC. The constraints on maximal buoy heave displacement is 0.5 $\mathrm{m}$. We can see that although the trajectories of state responses of system with different controllers are slightly different, the maximal heave displacement magnitudes are similar and the state constraint violation is avoided.

Fig. 10 shows the power output and energy output of system. The energy output without wave prediction is $19.58 \mathrm{~kJ}$ while the energy output with wave prediction horizons of $1 \mathrm{~s}, 2$ $\mathrm{s}$ and $3 \mathrm{~s}$ are $22.55 \mathrm{~kJ}, 24.36 \mathrm{~kJ}$ and $24.94 \mathrm{~kJ}$ respectively, which represents a $27 \%$ energy increase by incorporating wave prediction into optimal WEC control with a horizon of $3 \mathrm{~s}$. Fig. 10 shows that the noncausal optimal WEC control with wave predictions can help the WEC system to get a better energy conversion efficiency without increasing the state and control input magnitudes, i.e. introducing potential constraint violations.

Fig. 11 shows the energy output of the system with different

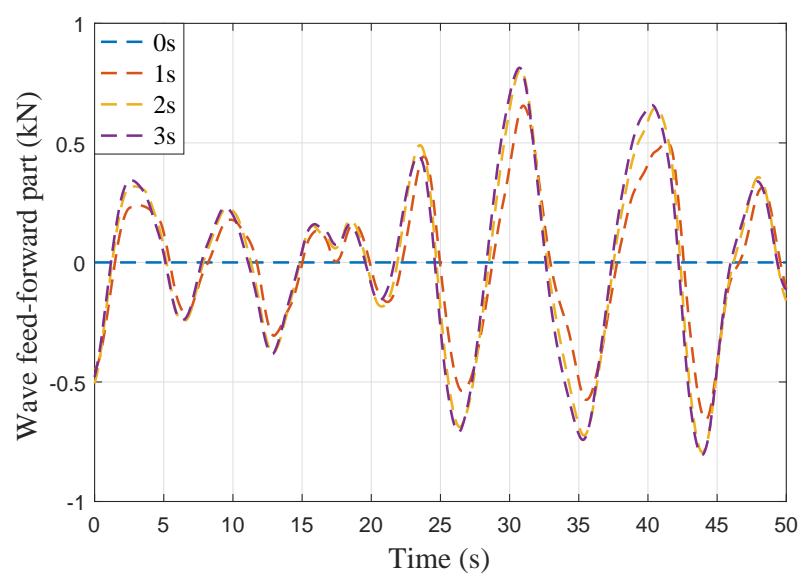

Fig. 8. Feedforward control input magnitude with different wave prediction horizons ( $0 \mathrm{~s}$ corresponds to no wave prediction).

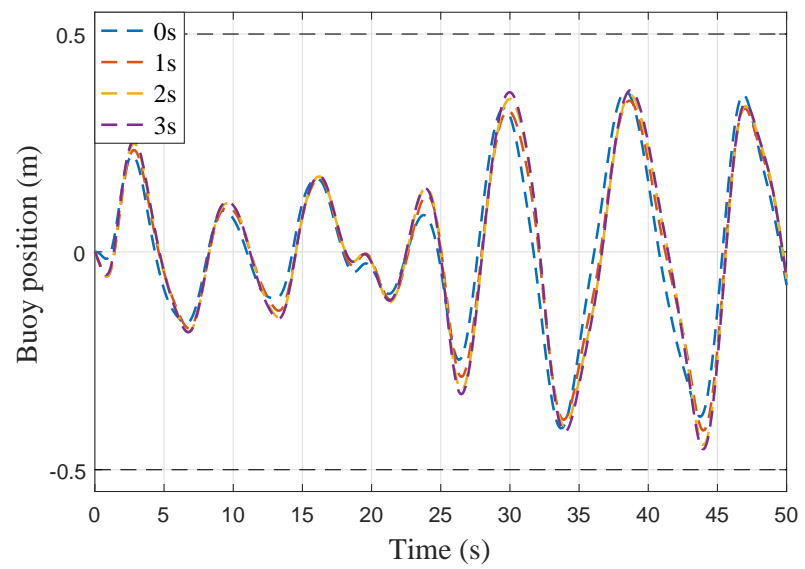

Fig. 9. Float heave displacement with different wave prediction horizons.

wave perdition horizons. We can see that the energy output increases significantly when the wave prediction horizon is within $3 \mathrm{~s}$. We also find that the WEC LOC with longer wave prediction has a better performance; however, the benefit of further increasing wave prediction horizon beyond $3 \mathrm{~s}$ becomes less obvious.

\section{B. The WEC model with parameters used in [13] with para- metric uncertainties}

To test the robustness of the proposed nonlinear LOC, we assume that the term $m_{\infty}$ from the radiation force (5) is incorrectly modeled at $100 \mathrm{~kg}$ instead of the true value 83.5 $\mathrm{kg}$ while the rest of the parameters remain the same as those used in section IV-A.

We tune the linear noncausal optimal controller using Algorithm 2 based on the inaccurate model with $m_{\infty}=100$ $\mathrm{kg}$. The controller weights in (12) are tuned to be $Q=$ $\operatorname{diag}(6,10,0, \ldots, 0)$ and $r=0.09$ such that the control input and state constraints are satisfied for the incorrect modeled system. The observer weights $R_{w}$ and $R_{v}$ remains the same. The $3 \mathrm{~s}$ wave prediction length is used. 

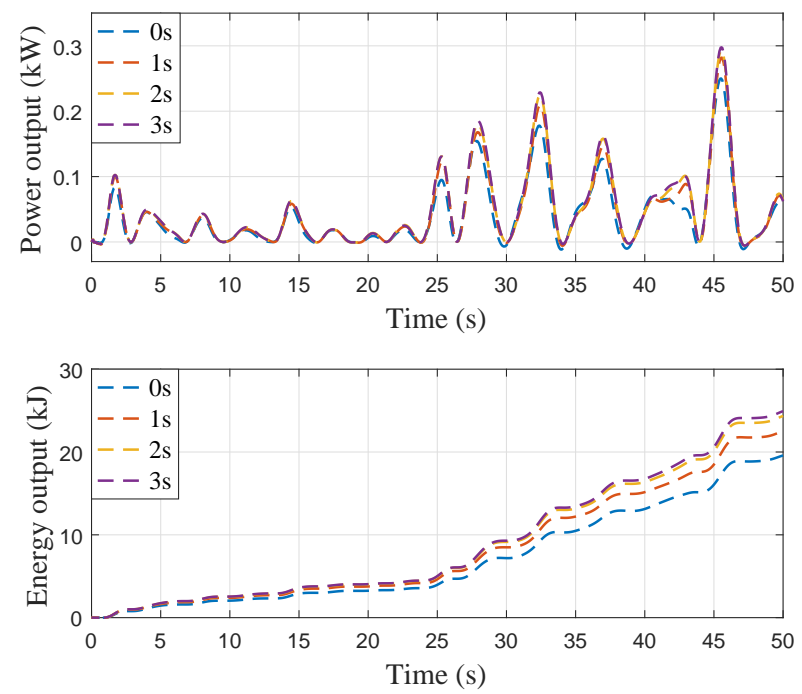

Fig. 10. Power output time simulation (a $27 \%$ efficiency increase between the linear causal control and the linear noncausal control with $3 \mathrm{~s}$ prediction horizon)

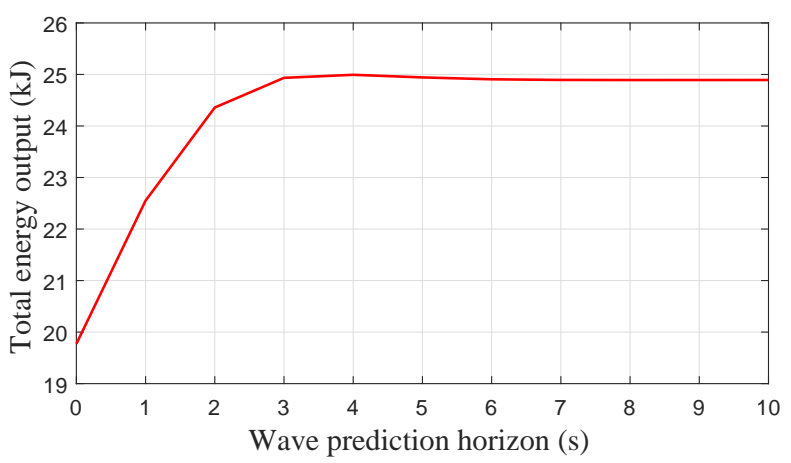

Fig. 11. Energy output with different wave predictions

After the offline computation of control coefficient $K_{x}$ and $K_{d}$ and Kalman gain $L$ using the incorrect model, we implement the observer with incorrect estimator updates (41) and the controller on the WEC model with $m_{\infty}=83.5 \mathrm{~kg}$.

Fig. 12 shows that the comparison of the control inputs and buoy position responses for the cases with and without parametric uncertainties. Note that in the simulation with parametric uncertainties, we use the inaccurate model for the designs of controller and observer. We can see that there are no significant differences between the two cases (41).

Fig. 13 shows the energy output. We can see that the energy output drops from $24.94 \mathrm{~kJ}$ to $24.52 \mathrm{~kJ}$ when the parametric uncertainties are taken into account. There is no significant performance degradation using the estimator and controller designed based on inaccurate model, which indicates that the linear noncausal optimal control method proposed in this paper has good robustness properties against model uncertainties.
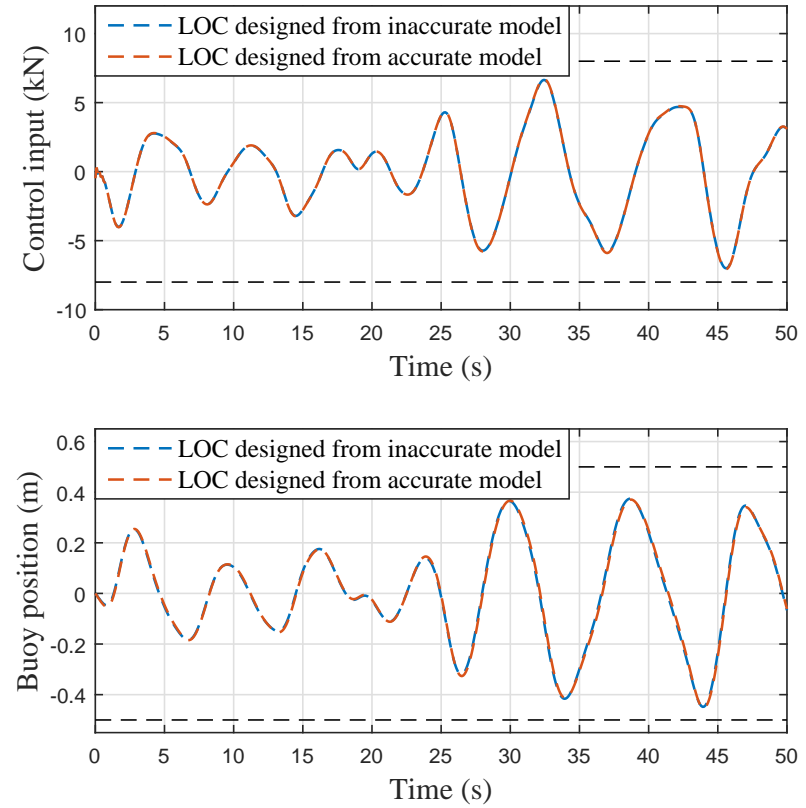

Fig. 12. Control input and buoy position magnititude of the WEC.
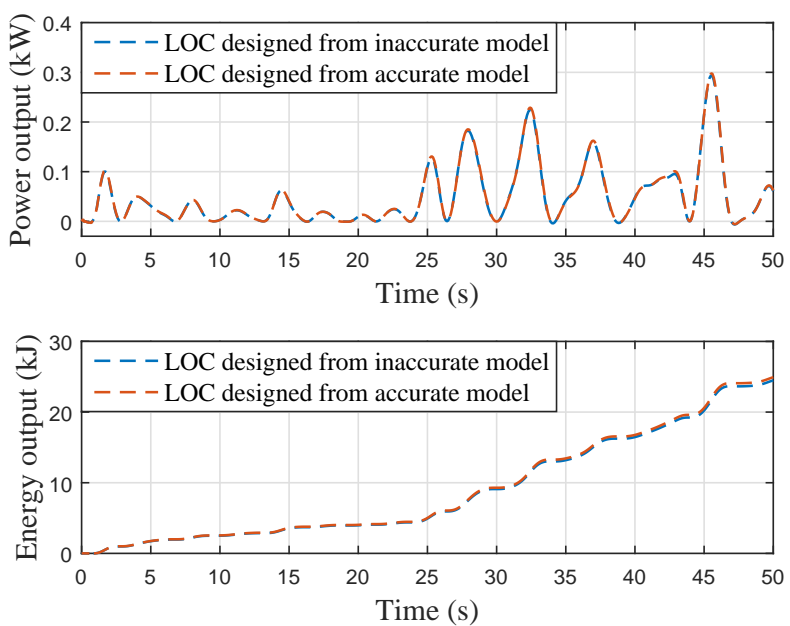

Fig. 13. Power and energy output of the WEC.

\section{Simulation comparison with a bigger WEC}

To show the universal efficacy of the proposed control strategy, we do simulations on a bigger WEC than that used in the last subsection. The parameters of this WEC model is shown in Table II.

By comparing Fig. 14 with Fig. 4, we can see that, the closed-loop system of WEC is less strongly stable compared with the WEC in section IV-A. By "less strongly stable", we mean that the eigenvalues of closed-loop system $A+B K_{x}$ are close to the unit circle, which result in $\Phi^{i}=\left(A+B K_{x}\right)^{i}$ decreasing slower than that in section IV-A with the increase of $i$. Such properties will have the following impacts.

1) The WEC linear noncausal optimal control requires a 
TABLE II

THE PARAMETERS USED FOR THE WEC MODEL IN SIMULATION SET B

\begin{tabular}{lll}
\hline Description & Notation & values \\
\hline Stiffness & $k_{s}$ & $6.39 \times 10^{5} \mathrm{~N} / \mathrm{m}$ \\
Float mass & $m_{s}$ & $7 \times 10^{4} \mathrm{~kg}$ \\
Added mass & $m_{a}$ & $1 \times 10^{4} \mathrm{~kg}$ \\
Total mass & $m$ & $8 \times 10^{4} \mathrm{~kg}$ \\
Input force limit & $u_{\max }$ & $80 \mathrm{kN}$ \\
Float heave limit & $\Phi_{\max }$ & $4 \mathrm{~m}$ \\
\hline
\end{tabular}
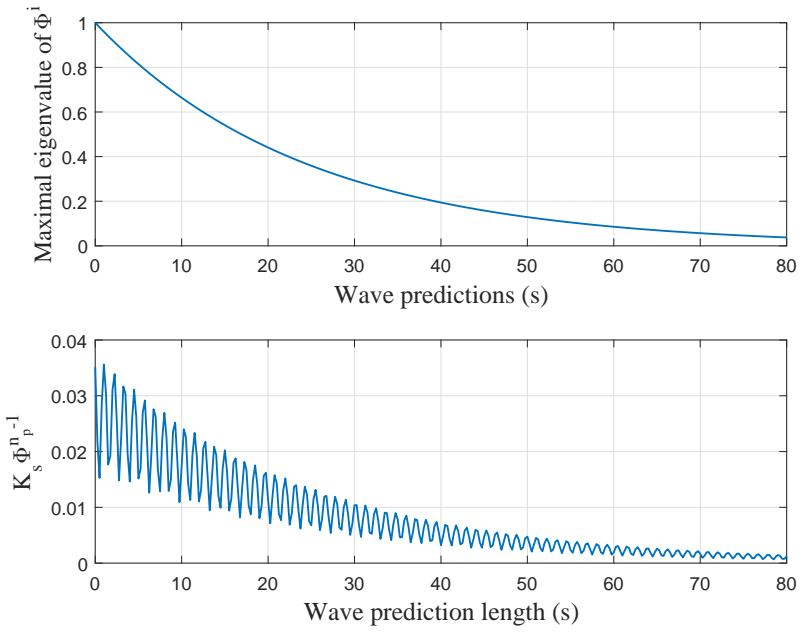

Fig. 14. The maximal eigenvalue of $\Phi^{i}$ decreases slower with the increase of $i$ compared with that of the smaller WEC in section IV-A.

longer prediction horizon for this term to be small enough and negligible.

2) The wave feedforward control part $K_{d} \mathbf{w}$ has a larger impact on the WEC performance.

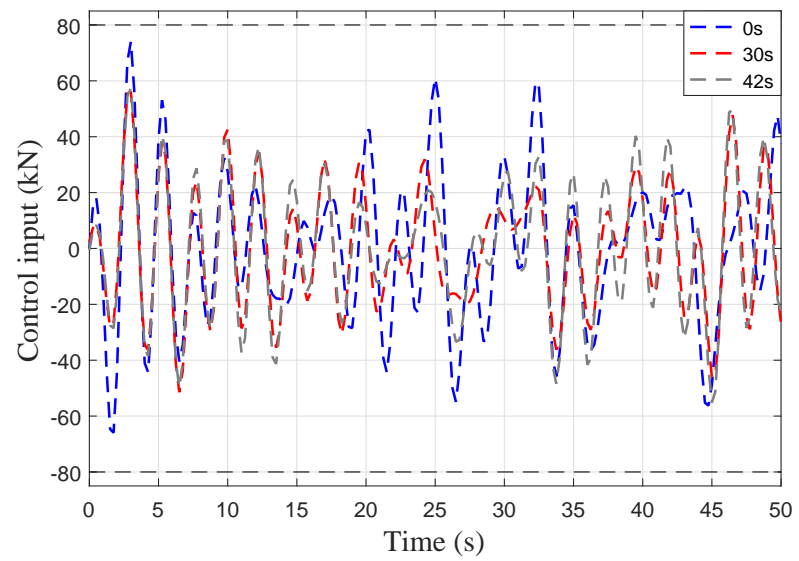

Fig. 15. Control input magnitude with different wave prediction horizons (0 $\mathrm{s}$ corresponds to no wave prediction).

Next, we present the time simulations of the WEC with designed linear noncausal optimal controller with the same wave profile used in section IV-A as shown in Fig. 6.

Three cases with different controllers are calculated with different wave prediction horizons (0 $\mathrm{s}, 30 \mathrm{~s}$ and $42 \mathrm{~s})$.

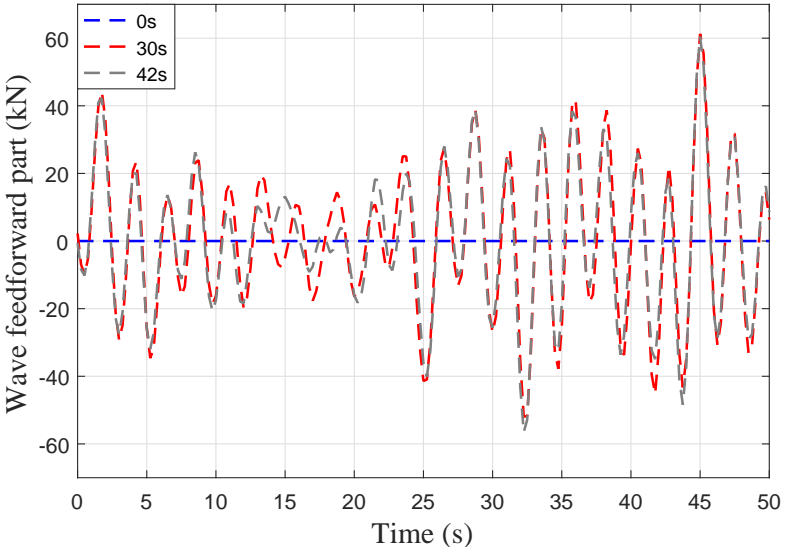

Fig. 16. Feedforward control input magnitude with different wave prediction horizons $(0 \mathrm{~s}$ corresponds to no wave prediction).

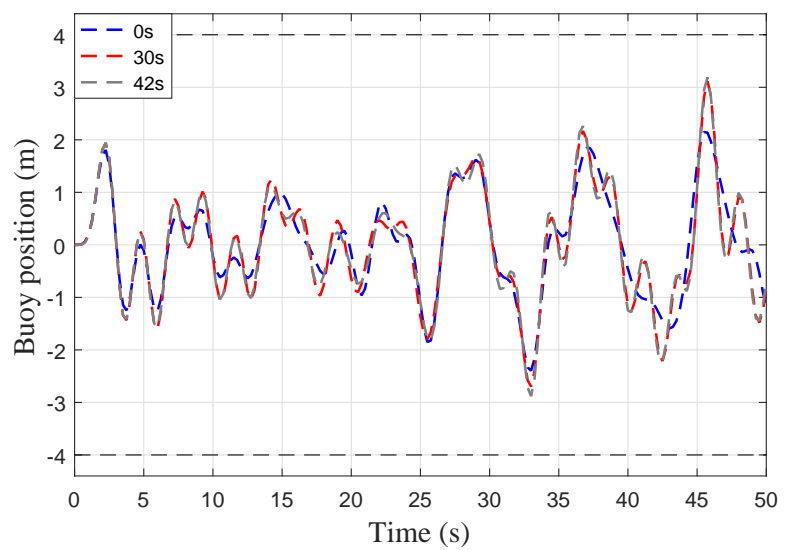

Fig. 17. Float heave displacement with different wave prediction horizons.

Figs. 15-17 show the control inputs $u=K_{x} x+K_{d} \mathbf{w}$, the feedforward control signals and buoy heave displacement for the three cases respectively.

By comparing Figs. 15-16 with Figs. 7-8, we can see that the the ratio of the maximum magnitude of the feedforward part (Fig. 16) to that of the total control (Fig. 15) is much bigger than the ratio for the smaller WEC case. This means the wave prediction plays a more important role in control signal in the less stable WEC design. Meanwhile, the maximal control input signals and maximal buoy heave displacement are similar over different prediction horizons.

Fig. 18 shows the power output and energy output of system. The energy output of system without wave prediction is 5575 $\mathrm{kJ}$ while the energy output of system with wave prediction of $30 \mathrm{~s}$ and $42 \mathrm{~s}$ are $6492 \mathrm{~kJ}$ and $7637 \mathrm{~kJ}$ respectively.

From Figs. 15-18, we can see that if a closed-loop system is less strongly stable, the benefits of incorporating wave predictions become greater, i.e $37 \%$ of energy conversion rate increase compared to $27 \%$, while the state and control input magnitudes are not changed significantly. However, to achieve better performance, much longer wave prediction horizons are required. 

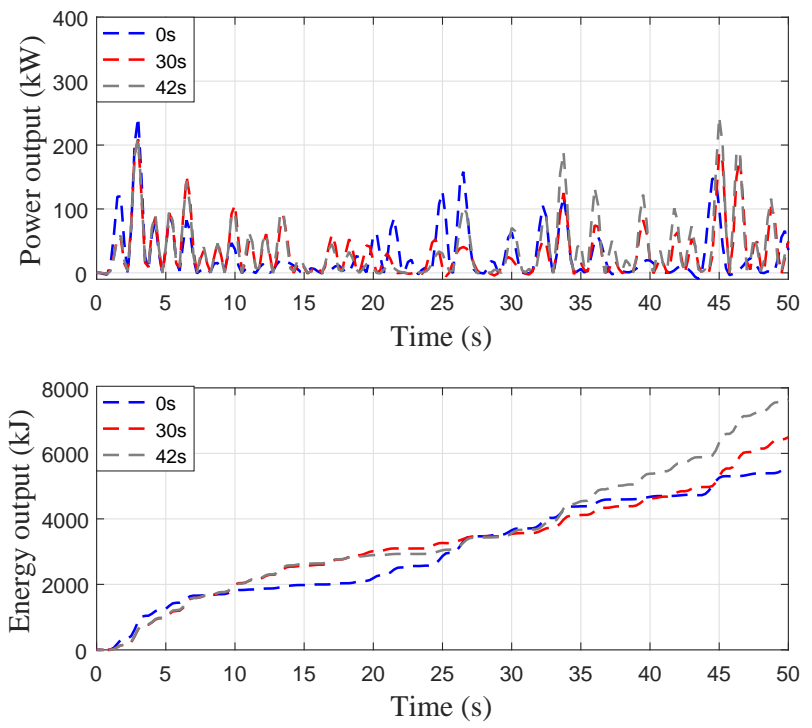

Fig. 18. Power output time simulation (a 37\% energy increase between the linear causal control and the linear noncausal control with 42 s prediction horizon)

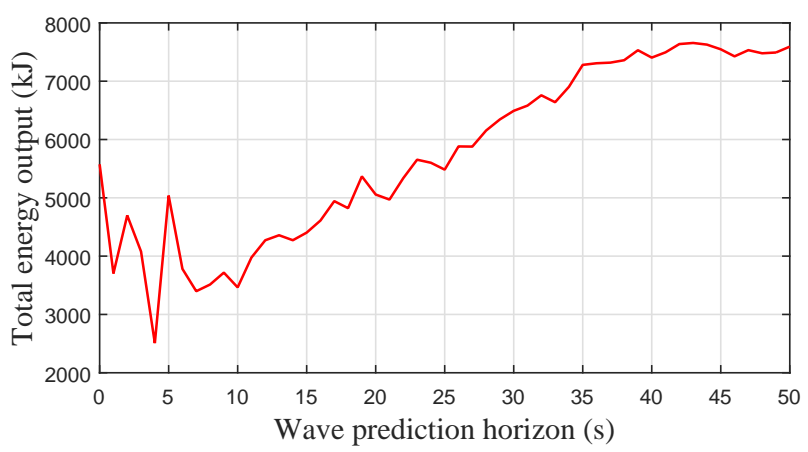

Fig. 19. Energy output with different wave predictions

Fig. 19 shows the energy output of system with different wave perdition horizons. We can see that the energy output keeps increasing until the wave prediction horizon reaches 40 s, which validates our analysis that for less stable systems, longer wave predictions are required and the impact of the wave prediction on systems' performance are greater. We can also find some fluctuation occurs between $0-10 \mathrm{~s}$ of the wave prediction horizons. This is because with an insufficient wave prediction length, as shown in Fig. 14, the term $K_{s} \Phi^{n_{p}-1} s_{n_{p}}$ is non-negligible so that Algorithm 2 with insufficient wave prediction horizons has a loss of optimality for the optimal control policy for WEC.

\section{CONCLUSions}

In this paper, we have developed a linear noncausal optimal control strategy for WECs. The optimal control strategy consists of a linear causal state feedback part and a linear anticausal feedforward part to incorporate the influence of wave prediction. This linear noncausal optimal controller can directly maximize the energy output with a sufficiently low computational load since the gains of the controller can be calculated offline. It is also easy to tune and find the trade-off between the energy output, robust stability and the satisfaction of constraints.

The paper also studies the impact of incoming wave predictions on the WEC LOC performance. The simulation shows that the feedforward part for incorporating wave prediction information can increase the systems performance without introducing the issue of constraint violation. The explicit analysis and demonstration of incoming wave prediction with different horizons provide a general guideline for finding the trade-off between extra cost caused by the requirement of wave predication and the LOC performance. The results of this paper also provide the basis to analyze the stability issues of model predictive control of WECs. In our future work, we will investigate the theoretical basis of the robustness of the proposed control against WEC dynamic uncertainties and wave prediction error.

\section{ACKNOWLEDGMENT}

The research was partially funded by Newton Mobility Grant (No. IE150833) and the Newton Advanced Fellowship (No. NA160436) from the Royal Society, UK. The authors gratefully acknowledge these supports.

\section{REFERENCES}

[1] S. H. Salter, "Wave power," Nature, vol. 249, no. 5459, pp. 720-724, 1974.

[2] J. Ringwood, G. Bacelli, and F. Fusco, "Energy-maximizing control of wave-energy converters: The development of control system technology to optimize their operation," Control Systems, IEEE, vol. 34, no. 5, pp. 30-55, 2014.

[3] J. Falnes, Ocean Waves and Oscillating Systems. Cambridge University Press, 2002

[4] A. Babarit and A. Clément, "Optimal latching control of a wave energy device in regular and irregular waves," Applied Ocean Research, vol. 28, no. 2, pp. 77-91, 2006.

[5] U. A. Korde, "Latching control of deep water wave energy devices using an active reference," Ocean engineering, vol. 29, no. 11, pp. 1343-1355, 2002.

[6] K. Budal, J. Falnes, T. Hals, L. Iversen, and T. Onshus, "Model experiment with a phase controlled point absorber," in Proceedings of Second International Symposium on Wave and Tidal Energy, 1981, pp. 191-206.

[7] A. Babarit, M. Guglielmi, and A. H. Clément, "Declutching control of a wave energy converter," Ocean Engineering, vol. 36, no. 12, pp. 10151024,2009

[8] J. Scruggs, S. Lattanzio, A. Taflanidis, and I. Cassidy, "Optimal causal control of a wave energy converter in a random sea," Applied Ocean Research, vol. 42, pp. 1-15, 2013.

[9] S. R. Nielsen, Q. Zhou, M. M. Kramer, B. Basu, and Z. Zhang, "Optimal control of nonlinear wave energy point converters," Ocean engineering, vol. 72, pp. 176-187, 2013.

[10] G. Li, G. Weiss, M. Mueller, S. Townley, and M. R. Belmont, "Wave energy converter control by wave prediction and dynamic programming," Renewable Energy, vol. 48, pp. 392-403, 2012.

[11] M. Belmont, J. Christmas, J. Dannenberg, T. Hilmer, J. Duncan, J. Duncan, and B. Ferrier, "An examination of the feasibility of linear deterministic sea wave prediction in multidirectional seas using wave profiling radar: Theory, simulation, and sea trials," Journal of Atmospheric and Oceanic Technology, vol. 31, no. 7, pp. 1601-1614, 2014.

[12] H. Santo, P. Taylor, E. C. Moreno, P. Stansby, R. E. Taylor, L. Sun, and J. Zang, "Extreme motion and response statistics for survival of the three-float wave energy converter $\mathrm{m} 4$ in intermediate water depth," Journal of Fluid Mechanics, vol. 813, pp. 175-204, 2017.

[13] Z. Yu and J. Falnes, "State-space modelling of a vertical cylinder in heave," Applied Ocean Research, vol. 17, no. 5, pp. 265-275, 1995. 
[14] S.-R. Huang, H.-T. Chen, C.-H. Chung, C.-Y. Chu, G.-C. Li, and C.C. Wu, "Multivariable direct-drive linear generators for wave energy," Applied energy, vol. 100, pp. 112-117, 2012.

[15] G. Weiss, G. Li, M. Mueller, S. Townley, and M. Belmont, "Optimal control of wave energy converters using deterministic sea wave prediction," Fuelling the Future: Advances in Science and Technologies for Energy Generation, Transmission and Storage, p. 396, 2012.

[16] R. Bellman, "The theory of dynamic programming," DTIC Document, Tech. Rep., 1954.

[17] C. Lee, "Wamit user manual," 1995.

[18] O. I. Olanrewaju and J. M. Maciejowski, "Implications of dissipativity on stability of economic model predictive controlthe indefinite linear quadratic case," Systems \& Control Letters, vol. 100, pp. 43-50, 2017.

[19] M. Zanon, S. Gros, and M. Diehl, "Indefinite linear mpc and approximated economic mpc for nonlinear systems," Journal of Process Control, vol. 24, no. 8, pp. 1273-1281, 2014.

[20] F. Fusco and J. V. Ringwood, "Short-term wave forecasting for real-time control of wave energy converters," IEEE Transactions on Sustainable Energy, vol. 1, no. 2, pp. 99-106, 2010.

[21] B. Kinsman, Wind waves: their generation and propagation on the ocean surface. Courier Corporation, 1965.

[22] M. J. Tucker and E. G. Pitt, Waves in ocean engineering, 2001, no. Volume 5.

[23] G. Li and M. R. Belmont, "Model predictive control of sea wave energy converters-part i: A convex approach for the case of a single device," Renewable Energy, vol. 69, pp. 453-463, 2014. 